\title{
Leben ist das größere Problem Philosophische Annäherung an eine Naturgeschichte der Freiheit
}

1. Freiheit im Widerstreit. Über die Freiheit wird derzeit wieder einmal viel gestritten. Schon von den antiken Denkern sind solche Debatten überliefert, die Philosophen des 17. und 18. Jahrhunderts haben sie fortgesetzt, und im 19. Jahrhundert herrschte der Glaube vor, den Streit endgültig erledigen zu können. Dass es dazu nicht gekommen ist, liegt auch an den Widersachern der Freiheit: Denn durch nichts wird die Freiheit besser unter Beweis gestellt, als durch den Versuch, sie zu bestreiten. Warum sollte es nötig sein, sie in Abrede zu stellen, und wie sollte das möglich sein, wenn es Freiheit nicht gäbe?

Der Versuch, sie zu bestreiten, könnte nur dann als „unfrei“ bezeichnet werden, wenn der Theoretiker zu seinen abschlägigen Thesen gezwungen worden wäre. Denkbar wäre auch, dass er unter Drogen stünde. Doch ich bin weit entfernt, mit einer despektierlichen Mutmaßung aufzuwarten. Vielmehr achte ich auch die Freiheit derer, die sie bestreiten, und wesentlich mit Blick auf die freiwilligen Gegner der Freiheit unternehme ich einen auf Anschaulichkeit angelegten Versuch, die Freiheit philosophisch so zu beschreiben, dass auch die lebenswissenschaftlichen Apostaten der Freiheit erkennen können, wie sehr die Freiheit sie nicht nur persönlich, sondern auch in ihrem eigenen Fach, vornehmlich in Physiologie und Biologie, berührt.

2. Die alltägliche Gegenwart der Freiheit. Freiheit, von der wir Menschen mit Rekurs auf unser Selbstbewusstsein sprechen, ist die Freiheit unseres eigenen Tuns. Wir wollen tun und lassen, was wir wollen, und wenn uns daran etwas hindert, sehen wir unsere Freiheit eingeschränkt. Die Hindernisse können verschieden sein, wie uns die Rede von der „Beinfreiheit“", die wir uns im Fond eines Wagens wünschen, die „Armfreiheit“, auf die wir bei der Anprobe eines Jacketts zu achten haben, oder die „Bewegungsfreiheit“ eines Kindes im Laufstall lehrt. Hindernisse anderer Art sind im Spiel, wenn von der „Reisefreiheit“ des Touristen, von der „Niederlassungsfreiheit" des Arztes oder von der „Meinungsfreiheit“" die Rede ist. 
Freiheit ist also ein Wort, das in vielen Lagen zur Anwendung kommt. Sogar in der Physik ist von den „Freiheitsgraden“ eines beweglichen Körpers die Rede. Aber die vielfältige Verwendung schließt nicht aus, dass es einen erkennbaren Ausgangspunkt für den weit gefächerten Gebrauch des Ausdrucks gibt.

Die Etymologie des Wortes verweist auf den gesellschaftlichen Handlungszusammenhang des Menschen. „Freiheit“ hängt in seiner Herkunft mit „Freund“ und „Frieden" zusammen; es bezeichnet bereits in einer frühen gotischen Verwendung den „Zustand der Freihalsigkeit". ${ }^{1}$ Dazu braucht man nur zu wissen, dass der Ring um den Hals das altgermanische Kennzeichen der Sklaven war. „Frei“ war einer, der nicht direkt dem Willen eines anderen unterstand und somit seinem eigenen Willen folgen konnte. Als „Freier“ ließ sich somit auch ein Mann bezeichnen, der eine heiratsfähige Frau aus der Verfügung der väterlichen Gewalt befreite.

3. Das individuelle Bewusstsein der Freiheit. Selbst wenn die Etymologie uns in die Irre führte, können wir ganz sicher sein, dass die Bedingung für eine sinnvolle Verwendung des Begriffs in der menschlichen Selbsterfahrung liegt. Wer nicht von sich aus weiß, was es heißt, von etwas frei zu kommen, in etwas frei zu sein oder aber eingesperrt zu werden, der wird wohl nie verstehen, was „Freiheit“ heißt. Zwar kann er beobachten, wie ein von der Leine losgelassener Hund seine Bewegungslust austobt; er wird auch eine Ahnung davon haben, warum Tiere im Zoo so traurig wirken und warum „Gefängnis“ als eine Strafe gilt; vermutlich wird er auch die Bilder von der Maueröffnung im Herbst 1989 nicht ohne Anteilnahme sehen. Deshalb ist nicht auszuschließen, dass er sich in einem Test zu einem weitgehend korrekten Gebrauch des Wortes „Freiheit“ als fähig erweist. Aber verstehen, was Freiheit bedeutet und warum sie dem Menschen so wichtig ist, wird er vermutlich nicht.

$\mathrm{Zu}$ diesem Verständnis gelangt man nur, indem man die Freiheit an sich selbst erfährt. Der letzte Schultag vor den Sommerferien, die Lust, ungestört im eigenen Zimmer zu sein, die Erlaubnis, aus dem Bücherschrank lesen zu dürfen, was immer man will, oder der erste Einkauf mit eigenem Geld: Das sind Situationen, in denen die Freiheit offenkundig ist. Auch der alljährlich wiederkehrende Kampf gegen die kratzigen Winterstrümpfe, gegen das elende „Leibchen“ und die viel zu langen „kurzen Hosen" ist in meiner Erinnerung mit echtem Freiheitsbewusstsein verbunden. Oder, viel später, das Gewicht der eigenen Entscheidung für die Philosophie und damit gegen den versammelten Rat der Familie.

Das sind mögliche Erfahrungen, die den ursprünglichen Sinn des Begriffs der Freiheit bestimmen. Es ist das aus eigenem Erleben stammende, individuelle Freiheits-

1 Kluge, Friedrich: Etymologisches Wörterbuch, 3. Aufl., Straßburg 1884. 
bewusstsein, das uns verstehen lässt, was Freiheit heißt. Es ist nicht zu sehen, warum dieses Bewusstsein im Widerspruch zu den Kausalrelationen der Natur stehen soll.

4. Kleine Phänomenologie der Freiheit. Die Selbsterfahrung der eigenen Freiheit dürfte eng mit dem Selbstvollzug unserer Lebendigkeit verbunden sein. Deshalb reichen ihre Wurzeln mit Sicherheit weit in die Naturgeschichte des Lebens zurück. Folglich ist es auch nicht abwegig, Analogien mit dem ungehinderten Lebensvollzug von Pflanzen und Tieren herzustellen. Sie werden von Spinozas umfassender Definition der Freiheit abgedeckt, ${ }^{2}$ passen aber auch zum weitläufigen Alltagsgebrauch des Begriffs. Es ist daher keineswegs vergeblich, sich an einer Naturgeschichte der Freiheit zu versuchen, die das, was der Mensch an sich selbst erfährt, in einen evolutionären Kontext stellt.

Zunächst aber ist festzuhalten, wie eng der skizzierte menschliche Erfahrungszusammenhang an das humane Selbstbewusstsein gebunden ist. Überall dort, wo sich das Individuum als Urheber seiner eigenen Bewegungen erlebt, wo es ihm gelingt, sich selbst aus einer unbequemen Lage zu befreien, wo es einem eigenen Wunsch nachgibt oder sich dem Verlangen eines anderen widersetzt, auch dort, wo es sich selbst bemühen, seine eigenen Kräfte einsetzen oder aus eigenem Impuls Ja oder Nein sagen kann: In allen diesen Fällen liegen Erfahrungen vor, die mit dem Bewusstsein der Freiheit verbunden sind.

Die Erfahrung der Selbstbewegung aus eigenem Impuls gewinnt an Prägnanz, sobald sie im Kontrast zu den Impulsen anderer steht. Das geschieht wesentlich durch die Artikulation seines Willens, in dem es den Impuls des eigenen Strebens für andere kenntlich zu machen sucht. Das Erleben der eigenen Freiheit ist mit der Ausübung des eigenen Wollens verknüpft. Dieses Wollen ist, wie Nietzsche sagt, auf ,etwas“ gerichtet. Aber das Selbstverständnis des Willens ist immer auch dadurch bestimmt, dass er sich im Verein mit und im Gegensatz zum Willen anderer zu behaupten hat. Wollen ist ausdrücklich eigenes Wollen, das sich in Relation zum Willen eines Gegenübers begreift.

Zwar wird man nachträglich auch dort, wo man ohne nachzudenken einfach seinen Eingebungen gefolgt ist, von Ungebundenheit und Freiheit sprechen. Andererseits kann man sich als extrem unfrei erfahren, wenn die Tür hinter einem ins Schloss gefallen ist, und der Weg zurück versperrt ist. Der vom Hochwasser Eingeschlos-

2 „Dasjenige Ding heißt frei, das aus der bloßen Notwendigkeit seiner Natur da ist und allein von sich zum Handeln bestimmt wird; notwendig aber oder vielmehr gezwungen (necessaria autem, vel potius coacta) dasjenige, was von einem anderen bestimmt wird, auf gewisse und bestimmte Weise zu sein und zu wirken." (Spinoza: Ethik, I, 7. Definition). 
sene, der vom Schnee Verschüttete, der vor Schreck Gelähmte wird sich in extremer Bedrängnis fühlen; niemand käme auf die Idee, ihn als frei zu begreifen, selbst wenn er noch über Handlungsalternativen verfügte. Hier ist es nicht der Willen eines anderen, der für die Einschränkung verantwortlich ist, sondern die Situation ist durch einen ungewöhnlichen Umstand derart verändert, dass die gewohnten Handlungschancen nicht gegeben sind.

Gleichwohl dürfte sich das Bewusstsein der menschlichen Freiheit wesentlich in jenen Lagen schärfen, in denen man sich gegen den Willen anderer zu behaupten hat. Das schließt nicht aus, dass man es als erhebend und befreiend empfindet, wenn man sich ohne Zwang dem Willen anderer anschließen kann. Aber dieses „ohne Zwang“, das für das Erleben der Freiheit grundlegend ist, kann nur in Relation zum Wollen anderer verstanden werden. Und da Unfreiheit mit Sicherheit dort gegeben ist, wo man unter dem Diktat des Willens eines anderen steht, darf man im Umkehrschluss behaupten, dass die eigene Freiheit sich im Vollzug des eigenen Willens entfaltet.

5. Freiheit im Gegeneinander der Willen. In ihrer artikulierten Form, so kann man den Ertrag der kleinen Phänomenologie resümieren, bringt die Freiheit einen gesellschaftlichen Tatbestand zum Ausdruck: Sie geht zwar von der Fähigkeit zur individuellen Selbstbewegung aus, setzt aber nicht nur die Kompetenz zum eigenen Handeln, sondern auch zur Verständigung über die eigenen Absichten voraus. Ferner unterstellt sie die Realität gegensätzlicher (und allemal auch individueller) Impulse. Denn nur im polaren Feld von Gegensatz und möglichem Einverständnis kann das eigene Wollen wirklich als frei oder unfrei erfahren werden.

Damit kann man die Essenz der Freiheit in der Befreiung von der Verfügung durch den Willen eines anderen sehen. Ursprünglich ist jedes Individuum dem Willen anderer unterworfen. In der Regel sind es die Eltern, die das Kind in ihrer Obhut haben und nach ihrer eigenen Einsicht mit ihm verfahren. Doch die organische Eigenständigkeit des Einzelnen greift in einer normalen Entwicklung sukzessive auf den Bewegungsapparat und den Ausdruck über. Das Individuum kann, muss und will sich aus eigenem Antrieb bewegen und hat sich zunehmend eigenständig zu artikulieren. So entsteht und wächst die Geschicklichkeit im Umgang mit sich selbst, die eine weitreichende Kontrolle auch sozial gerichteter Äußerungen im Gefolge hat.

Zugleich wachsen die Eigeninteressen und rufen unvermeidlich Konflikte hervor. Sie sind es, in denen sich der Wille des Einzelnen schärft. Im trotzigen „Nein“ des Kindes wird er ihm selbst und anderen vermutlich zuerst bewusst. Sobald er sich differenzierter äußern kann, wird der Wille zu einem für einen selbst wie für die anderen erkennbaren Movens der Freiheit, die ihr sicherstes Bewusstsein in der Unabhängigkeit vom Willen eines anderen hat. 
Die Eigenständigkeit des eigenen Wollens ist es somit, die wir meinen, wenn wir von Freiheit sprechen. Solange sich ein Mensch nach seinen eigenen Einsichten richten, solange er nach seinen eigenen Gründen handeln kann, begreift er sich als frei. Und daran ändert sich nichts, wenn er die Natur sowohl im Ganzen wie auch im Detail als „,determiniert“ bezeichnet. Die „Kausalität aus Freiheit“, von der man im Anschluss an Kant bis heute spricht, bezieht sich auf die Urheberschaft für das eigene Tun. Deren Subjekt ist das sich artikulierende „Selbst“ und damit das sich seiner selbst bewusste Ich, das keinen Anhaltspunkt dafür gibt, die Kausalität des Individuums könne zur Kausalität der Natur in Widerspruch stehen.

Die Zuschreibung der Urheberschaft erfolgt übrigens nicht mit Blick auf die Hand, die den Backenstreich tut. Die Freiheit ist nicht auf die Lippen beschränkt, über die das unbedachte Wort gegangen ist, und meint, selbst wenn sich jemand aus Ärger über sein Tun an die Stirne fasst, niemals bloß seinen Kopf oder das, was darinnen ist. Sie ist vielmehr stets auf den ganzen Menschen gerichtet, und zwar auf die Einheit, die er in seinem Empfinden, Erleben und Handeln selbst erfährt, die aber auch von seinesgleichen wahrgenommen und angesprochen wird.

Die organische Einheit eines Lebewesens trägt auch den praktischen, semantischen und symbolischen Konnex seiner Bewegungen. Die Einheit in der Wirkung und im Sinn seiner Äußerungen hat ihren Grund in der organischen Selbstbezüglichkeit des lebendigen Wesens, bei dem alles, was aus eigenen Systembedingungen heraus erfolgt, als Funktion eben dieses Systems angesehen werden muss. Noch dessen Offenheit und Veränderbarkeit stehen im Interesse des Systems. Folglich kann, ja, muss man auch die affektiven und intelligiblen Leistungen eines Organismus als Momente im Vollzug seiner Eigenart verstehen. Alles, was ihn daran hindert, nach seiner Eigenart zu agieren und zu reagieren, schränkt ihn derart ein, dass man von der Behinderung seines Lebensvollzugs sprechen kann. Das wäre ein Zustand, in dem man das Lebewesen nicht mehr als „frei“ bezeichnen könnte.

Doch lassen wir zunächst noch offen, ob sich der Begriff der Freiheit auch auf die ungehinderte Entfaltung nicht-menschlicher Lebewesen beziehen lässt. Beim Menschen ist es so, dass wir von Freiheit dort sprechen, wo er sich als Ganzer uneingeschränkt zum Ausdruck bringen kann. Dabei findet sie ihre Grenze an der Freiheit anderer, nicht aber an der Natur, erst recht nicht am Gehirn des Menschen, selbst wenn es so determiniert oder so determinierend wäre, wie es die Abtrünnigen der Freiheit, die ihre Freiheit nutzen, um Freiheit zu bestreiten, glauben.

Das hier skizzierte Verständnis von Freiheit hat eine lange Tradition. Jean Bodin hat ihm zu einem klassischen Ausdruck verholfen: „Natürliche Freiheit bedeutet für uns, ... keinem lebenden Menschen unterworfen zu sein und von niemand anderem 
Befehle entgegennehmen zu haben als von sich selbst, d. h. von der eigenen Vernunft, die stets im Einklang mit dem Willen Gottes steht. ${ }^{\text {“3 }}$

6. Freiheit nur unter der Gesetzmäßigkeit der Natur. Auch wenn im Gebrauch des ursprünglich auf den handelnden Menschen bezogenen Freiheitsbegriffs die Kausalität der Naturereignisse gar kein Thema ist, bleibt es eine unausweichliche Frage, wie denn der Mensch mit seinem ihn als Einheit auszeichnenden Freiheitsbewusstsein in den Zusammenhang jener Naturvorgänge passt, denen er selbst als Naturwesen zugehört und die er durch den Begriff der Kausalität zu erfassen sucht.

Die erste und wichtigste Auskunft ist die, dass der Mensch in seinem Freiheitsbewusstsein auf nichts so sehr angewiesen ist, wie auf die Verlässlichkeit der ihm bekannten Natur. Bei jedem Schritt, den er tut, bei jedem Bissen, den er schluckt, bei jedem Werkzeug, das er einsetzt, und bei jedem Haus, das er baut, vertraut er auf die unverbrüchliche Gesetzmäßigkeit der umgebenden Welt. Mag er die strikte, alles Geschehen tragende Geltung des Gesetzes der Kausalität auch noch so spät entdeckt und beschrieben haben: In der Sache gründet er seinen Umgang mit den Dingen schon immer auf die lückenlose Geltung der Naturgesetzlichkeit.

Wie hätte der Mensch Waffen ohne die Annahme herstellen können, dass sie immer auf dieselbe Weise wirken und durchschnittlich nur den verletzen, gegen den sie gerichtet sind? Wie hätten die Menschen je das Feuer domestizieren können, wenn sie hätten befürchten müssen, dass es jederzeit auch auf die Steine, die Erde, den Ofen oder das Löschwasser übergreift? Was hätten ihnen das Lernen und die Wissenschaft gebracht, wenn die Natur wirklich voller Lücken wäre, die sich erst dadurch füllen, dass einer sie mit freien Handlungen auszustopfen sucht?

Im Wissen und im handwerklichen Tun, im Planen und Erinnern, im Umgang mit dem eigenen Körper und den Gegenständen der äußeren Welt, seien es die gebrannten Ziegel, das Saatgut oder das Vieh: In alledem geht der Mensch von der Regelmäßigkeit des Naturgeschehens aus. Er nimmt sie als Voraussetzung seines Handelns an, setzt sie nach Art eines Mittels ein und kann gar nicht umhin, sie auch noch jenen Zuständen zu unterstellen, die er mit seinen willentlichen Aktivitäten erreichen will. Warum denn sollte er selbst eine Ausnahme von den Naturerscheinungen sein? Oder wird er es schon dadurch, dass er sich zur Ausnahme erklärt?

3 Bodin, Jean: Sechs Bücher über den Staat (1572), Buch I, Abschnitt 3. Dass Bodin nicht nur an die Übereinstimmung der eigenen Tat mit dem eigenen Willen, sondern auch an eine Koinzidenz mit dem Willen Gottes denkt, bringt den Anspruch auf die Vernunft im eigenen Willen zum Ausdruck. Wenn der Einzelne mit seinem Wollen nicht nur im Augenblick übereinstimmen will, muss er sich auf einsichtige Gründe stützen, deren Angemessenheit im Horizont seiner Selbst- und Weltkenntnis tatsächlich am besten dadurch angezeigt werden kann, dass man glauben darf, sie entsprächen dem Willen Gottes. 
Zur Ausnahme macht sich der Mensch jedenfalls noch nicht, wenn er anderen absichtlich ein Zeichen für das gibt, was geschehen oder verhindert werden soll. Auch andere Lebewesen geben sich Zeichen, die Reaktionen, wie gemeinsame Aufmerksamkeit oder Flucht, veranlassen. Auch hier sind gleich bleibende Ursachen und Wirkungen nicht nur tatsächlich gegeben, sondern auch von den reagierenden Wesen unterstellt. Nicht nur in der Verständigung über gemeinsame Reaktionen, sondern auch in der Täuschung von Feinden sind Regelmäßigkeiten habitualisiert, die kenntlich machen, dass die Funktion des Organismus auf der Gesetzmäßigkeit der Naturprozesse basiert. Deshalb geht man nicht zu weit, wenn man behauptet, dass Freiheit nur möglich ist, wo sich der Mensch auf die lückenlose Kausalität der Natur verlassen kann. Diese Behauptung gilt im Übrigen nicht nur für die äußeren Handlungskonditionen, sondern auch für die Vorgänge im Inneren des Organismus. Jeder Mensch, der isst und trinkt, weiß davon, und wer eine Kopfschmerztablette nimmt, glaubt daran. Mehr noch: Er müsste an sich selber irre werden, wenn seine Kausalität aus Freiheit im Widerspruch zur Kausalität der physischen und physiologischen - und am Ende natürlich auch - der neuronalen Prozesse stünde.

7. Die Natur im Widerstreit mit sich selbst. Die Natur, die wir sind, die wir erleben und die wir in separierten begrifflichen Leistungen erkennen, ist kein linearer Prozess im ungehinderten Übergang von Ursachen zu Wirkungen. Sie ist zunächst und in allem ein unendlich vielfältiges, myriadenhaft individuiertes und organisiertes, in labilen Gleichgewichten nur zeitweilig austariertes, aber fortlaufend durch sich selbst gestörtes Konglomerat von Gegensätzen, das überdies in seiner energetischen Grundstruktur auf Quanten beruht, deren Verhalten sich unter keinen Bedingungen berechnen lässt.

Vermutlich ginge man schon zu weit, wenn man von einem „System“ repulsiver und attraktiver, antagonistischer und symbiotischer Kräfte spräche. Aber es kommt unserem Verlangen, selbst Einheit zu sein, entgegen, wenn wir auch unser Gegenüber als Einheit begreifen. Und die Freiheit, die mit unserem Einheitsverlangen auf das Engste verbunden ist, schreiben wir auch diesem Gegenüber zu. Daher die Neigung, die Natur als Ganze selbst als lebendig und eigenständig anzusehen und sie begrifflich entsprechend auszuzeichnen.

Gleichwohl verzichte ich darauf, einen Begriff für das Flimmern der Energien zu finden, das sich uns als Natur zu erkennen gibt. Sicher ist nur, dass wir viel zu wenig sagen, wenn wir die kausale Ordnung exponieren, ohne hinzuzufügen, dass sich die Kausalität offenbar bestens mit der inkommensurablen Vielfalt und den chaotischen Gegensätzen in ihrer realen Bewegung verträgt. Erst die Kausalität ermöglicht die Bildung und Verstärkung einzelner Kräfte, und es schmälert ihre Gesetzlichkeit keineswegs, dass sie es ist, die alles wieder zerstört. 
Das gilt vor allem für die belebte Natur. Hier konzentrieren und multiplizieren sich die Gegensätze ins Unabsehbare. Hier bilden sich immer neue Einheiten, die Lebensformen zerstören und schaffen, welche ihrerseits neuartige Widerstände stimulieren. Am selben Individuum und in derselben Gattung gibt es einen rhythmischen Wechsel von Steigerung und Verfall. Unablässig werden Einheiten gebildet und wieder vernichtet, einzelne Wesen entstehen und vergehen, wobei es immer wieder vorkommt, dass sich völlig neue Arten ergeben.

In diesem synergetischen Feld fortgesetzter Kongruenzen und Oppositionen treten also lebendige Wesen auf, von denen sich, ohne dass es jemand bestreitet, sagen lässt, dass sie sich „aus eigenem Antrieb“ bewegen. Auch wenn ihre Bewegungen auf Naturgesetzen beruhen und obgleich sie durch und durch aus lückenlos aneinander liegenden Stoffen bestehen, die selbst ihrer spezifischen Gesetzmäßigkeit folgen, haben die lebendigen Wesen dennoch ihre eigene Dynamik, die sich nach ihren gattungsspezifischen Strukturen und nach Maßgabe ihrer Lernprozesse vollzieht. Angesichts der von den Apostaten der Freiheit unterstellten These einer durchgängig durch Kausalität festgelegten Natur ist das ein höchst unwahrscheinlicher, vielleicht sogar unmöglicher Tatbestand.

Und dennoch gibt es ihn. Er manifestiert sich in jedem Akt des Lebens, das dieselben Theoretiker, die Freiheit bestreiten, als ,spontan“ verursacht und als ,eigengesetzlich“ beschreiben. ${ }^{4}$ Wenn es aber spontane Bewegung, eigene Dynamik und sich selbst erhaltende Strukturen gibt, dann ist nicht einzusehen, warum es ausgerechnet die Freiheit nicht geben soll, die diese Ursprünglichkeit und Eigengesetzlichkeit eines lebendigen Wesens zum Ausdruck bringt.

8. Das Beispiel eines Schmetterlings. Es scheint niemanden zu wundern, dass die Kausalität der Natur eine so große Vielfalt an Formen hervorzubringen vermag und mit ihnen zahllose Wesen, die sich wechselseitig ihre Existenz streitig machen. Ehe wir uns über die Freiheit wundern, sollten wir darüber staunen, dass die Naturkausalität überhaupt so etwas wie Leben zulässt.

Möglich ist das Leben nur, weil die Natur nicht in geschlossener Front marschiert. Sie wird nicht von einer einzigen Kausalkette gezogen, sondern besteht auf einer Vielzahl sich wechselseitig verstärkender, behindernder und vernichtender Kräfte, und es ist das Wechselspiel dieser Kräfte, das zum Aufbau organischer Einheiten so genutzt wird, dass sie sich selbst als eine Kraft behaupten kann, die durch die Integration widerstreitender Kräfte entsteht und alles andere als eindeutig berechenbar

4 Roth, Gerhard: Gehirn und Selbstorganisation. In: Krohn, W. \& G. Küppers (Hg.), Selbstorganisation. Aspekte einer wissenschaftlichen Revolution, Braunschweig, Wiesbaden 1990, S. 167-180. 
ist. Angesichts der Vielfalt tatsächlich wirkender Kräfte ist es, trotz strikter Determination, noch nicht einmal möglich, die nächstliegende Wirkung vorherzusehen. ${ }^{5}$

Vielleicht kann man das an einem Beispiel illustrieren: Bei einem Schmetterling erfolgt der kontingente Richtungswechsel in Bruchteilen von Sekunden. Jede seiner ausgeführten Bewegungen dürfte kausal verursacht und dennoch vorab kaum berechenbar sein, weil viel zu viele vorher gar nicht absehbare Kräfte von außen auf den Organismus und (teils in Reaktion darauf, teils aus eigenen Strukturbedingungen) in ihm selber wirken. Jede Analyse der Bewegung des Schmetterlings hat mit der Vielzahl von inneren und äußeren Ursachen zu rechnen, deren Wirkungen in der Bewegung des Insekts zwar eindeutig sind, aber dennoch schwer vorausberechnet werden können.

Das kann nur gelingen, wenn man die Ganzheit des Organismus ins Kalkül zieht: Der Organismus ist das „System“, das viele Kräfte auf die für das Ganze charakteristische Weise vermittelt. Dabei verfährt es in den einzelnen Akten kausal, aber die Kausalität des Systems, die sich in den realen Bewegungen des Schmetterlings zeigt, kann nicht auf ein lineares Ursache-Wirkungsschema reduziert werden, weil viele Ursachen gegenläufig ineinanderwirken. ${ }^{6}$ Dadurch, dass sich das System als Ganzes „verhält", kann es seine eigenen Bewegungsmuster reproduzieren. Und solange dem System keine Gewalt angetan wird, bewegt es sich nach seinem eigenen Gesetz. Wenn ein menschliches Wesen dieser Eigengesetzlichkeit folgt, nennen wir es „,frei“. Warum sollte man diese Redeweise nicht auf andere Lebewesen übertragen können? Ein Schmetterling im Netz ist nicht mehr frei zu nennen, aber solange er ungehindert über Wiesen und Hecken fliegt, sehr wohl.

9. Eigenes im Wechselspiel der Kräfte. Ich stelle erneut die für das Verständnis von Freiheit entscheidende, aber in der Regel vergessene Frage: Wie können unter den lückenlos wirksamen kausalen Kräften der Natur überhaupt die Bewegungs-

\footnotetext{
In der nüchternen Sprache von Andreas Herz: „Die Wahrscheinlichkeiten für das Eintreten eines Ereignisses gehorchen dabei zwar deterministischen Gesetzen; ob das Ereignis jedoch auch eintritt, und wann dies geschieht, ist unvorhersagbar. Zusätzlich stellt ein Organismus kein abgeschlossenes System dar, sondern steht andauernd mit seiner beliebig hochdimensionalen Umwelt in Verbindung. Aus beiden Gründen kann die zukünftige Entwicklung eines Organismus selbst bei vollständig bekannten internen Anfangsbedingungen nicht exakt vorausgesagt werden.“ (s. Neuronaler Determinismus: Nur eine Illusion? In: Heilinger, Jan-Christoph (Hg.), Naturgeschichte der Freiheit, Berlin, New York: de Gruyter, 2007, S. 35).

6 Von einer näheren Betrachtung der nicht eindeutig determinierten offenen Systeme organischer Wesen sehe ich hier ab und verweise auf den Beitrag von Andreas V. M. Herz, Neuronaler Determinismus und Naturwissenschaft (wie Anm. 5).
} 
spielräume entstehen, die sich im Sinn der Freiheit deuten lassen? Die Antwort erfordert eine Reflexion auf die Natur, deren Mechanik es offenbar nicht verhindert, dass Leben entsteht.

Schon diese Art zu reden könnte anstößig klingen, weil es doch die Mechanik der Natur sein muss, die Leben möglich macht. Wie anders sollte denn Leben entstanden sein, als unter den Konditionen unausgesetzter Kausalität? Die Frage aber ist, wie die strikte Folge von Ursachen und Wirkungen dazu führen kann, dass sich inmitten der Mechanik der Natur separierte Kräfte und Kraftzentren bilden, die ihre eigene Ordnung haben, um ihr entsprechend zu selegieren und zu reagieren. ${ }^{7}$ Dass eine Amöbe ihre eigenen Rezeptions- und Reaktionsformen ausprägt, ist angesichts der Uniformität des Kausalitätsprinzips ein erstaunlicher Tatbestand.

Nicht weniger verwunderlich ist die Fähigkeit höher organisierter Lebewesen, nicht nur spezifische, sondern auch individuelle Verhaltensformen auszubilden. Sie leben im selben Umfeld und haben den gleichen Aufbau, können sich aber dennoch unterschiedlich verhalten. Sie können Spielräume nutzen und auf veränderte Lagen mit veränderten Programmen reagieren. Eben darin zeigt sich die Eigenart des Lebens, das Organismen schafft, welche die Fähigkeit auszeichnet, sich aus eigenem Impuls und in eigener Dynamik zu erhalten. Das Leben hat seine Eigenart darin, dass es Systeme schafft, die nach ihrer eigenen Gesetzmäßigkeit verfahren. Die Aussage gilt sowohl für einzelne Wesen als auch für die Populationen, in denen sie möglich sind.

Allein die Tatsache, dass die Individualität eines Lebewesens nicht nur in seiner Gestalt, sondern auch in seinen Verhaltensformen zum Ausdruck kommt, beweist einen Spielraum der lebendigen Natur, der nicht entstehen könnte, wenn es nur die lineare Kausalität der mechanischen Kräfte gäbe. Tatsächlich aber herrscht der Widerstreit unablässig aufeinander einwirkender Kräfte, die sich stören und verstärken, aufheben und neu entstehen, in Gleichgewichtslagen binden und wieder daraus lösen lassen. Es gibt die systemisch geordneten Wirkungsformen lebendiger Wesen, die jeder Art zu ihrer spezifischen und jedem Organismus zu seiner individuellen Form des Verhaltens verhilft. Dadurch sind eigene Äußerungsweisen möglich. Wo es aber Eigenes gibt, kann es von anderem gefördert, eingeschränkt oder beseitigt werden. Das entspricht den Wirkungsformen, die wir aus dem Gebrauch der menschlichen Freiheit kennen - auch und gerade dort, wo sie behindert wird.

7 Julian Nida-Rümelin bietet dafür eine verblüffend einfache Erklärung, indem er in seinem Kugelexperiment demonstriert, dass selbst eine strikt kausale Naturordnung nicht alle Ereignisfolgen festlegt (s. Nida-Rümelin, Julian: Über menschliche Freiheit, Stuttgart 2005, § 5; ders.: Strukturelle Rationalität, Stuttgart 2001, Kapitel 2). 
Wer nun die Besonderheiten einer Gattung oder eines Lebewesens mit der Kausalität der Natur für vereinbar hält, der hat nicht länger Grund, einen Widerspruch zwischen Kausalität und Freiheit anzunehmen. Denn Freiheit ist der prozessuale Ausdruck der Eigentümlichkeit eines menschlichen Wesens - in Relation zur Eigentümlichkeit von seinesgleichen. Sollte es gelingen, dieses Verständnis von Freiheit plausibel zu machen, böte es die Chance, Vorformen der Freiheit auch bei anderen lebendigen Wesen kenntlich zu machen. Das entspricht der Wahrnehmung durch den unbefangen urteilenden Menschen, der Freiheit (als die nicht behinderte Spontaneität seines eigenen Selbst) im strengen Sinn zwar nur von sich selber kennt, in der lebendigen Natur aber dennoch das ihm Verwandte empfindet. So war es Leibniz und Kant, Goethe und Alexander von Humboldt möglich, die ,freie Natur" des Lebens zu bewundern, die sie von der „gefesselten Natur" rein mechanischer Prozesse zu unterscheiden wussten. ${ }^{8}$

10. Der natürliche Sockel der Freiheit. „Freiheit“ bezeichnet die ungehinderte Entfaltung einer lebendigen Bewegung nach den Konditionen, die für das Lebewesen spezifisch sind. Beim Mensch liegt diese Kondition im eigenen Willen und in der eigenen Einsicht. Beide treten in der Regel als Anwälte der organischen Eigenständigkeit des menschlichen Wesens auf. Auch jener Akt, in dem sie verschwinden, nämlich im gelingenden Suizid, kann noch als freier Akt der Sicherung der personalen Integrität begriffen werden. In diesem Fall liegt die Eigenständigkeit im Bewusstsein sozialer und moralischer Verbindlichkeiten, mit denen sich eine Person identifiziert. Also kann sie selbst noch in einer aussichtslosen Lage, in der ihr kein Handlungsspielraum mehr zu bleiben scheint, den Akt der Selbsttötung als frei begreifen.

Sehen wir von der Grenzsituation der Selbsttötung ab, dann liegt die Freiheit des Menschen in der ungehinderten Eigenständigkeit einer Selbstbewegung, die auf die Erhaltung des Lebens bezogen ist. Die Instanz, welche diese Einheit wahrt, kann als Bewusstsein bezeichnet werden, dass seinerseits Instanzen und Instrumentarien zur Regulierung und Steuerung des Verhaltens in einem durch Wissen und soziale Verbindlichkeiten strukturierten Umfeld ausbildet. Vernunft, Einsicht oder Wille sind solche Instanzen und Instrumentarien. Der übliche Begriffsgebrauch von Freiheit ist auf ihren Einsatz, kurz: auf die intelligiblen Fähigkeiten des Menschen bezogen. Es gibt keinen Anlass, daran etwas zu ändern. Gleichwohl kann es das Verständnis

8 Dazu Kaulbach, Friedrich: Philosophie der Bewegung, Köln 1965, S. 23ff.; ders.: Philosophie der Beschreibung, Köln, Graz 1968, S. 62ff.; ferner: Hamel, Jürgen, Knobloch, Eberhard \& Herbert Pieper (Hg.): Alexander von Humboldt in Berlin. Sein Einfluß auf die Entwicklung der Wissenschaften. Beiträge zu einem Symposium, Augsburg 2003. 
dieses Begriffs von Freiheit erhellen, wenn wir seinen Geltungsbereich versuchsweise auf das Verhalten von Lebewesen übertragen, die vermutlich nicht über Vernunft, Einsicht und Wille verfügen.

Jedermann weiß, dass in den Freiheitsimpuls des Menschen auch andere Momente eingehen können. Ohne Antriebe, Empfindungen, Gefühle und Gewohnheiten hätte es keinen Sinn von Freiheit zu sprechen. Oft vollstreckt der Wille nur, was die Affekte fordern. Doch auch dann ist er nicht unfrei. Zwar entspricht es dem Ideal, möglichst nur nach eigener Einsicht zu entscheiden, aber im Einzelfall muss jede menschliche Handlung, die von anderen nicht erzwungen ist, als „frei“ bezeichnet werden. ${ }^{9}$ In ihr folgt der Mensch seinem eigenen Impuls - bricht auf, wohin er will, liebt die Frau, die ihm gefällt, oder kauft Dinge, die er gar nicht braucht.

Wenn es dem üblichen Sprachgebrauch nicht entgegensteht, auch in diesen Fällen menschlichen Verhaltens von Freiheit zu sprechen, und wenn überdies auch noch darauf verwiesen werden kann, dass diese Rede als durchaus konsequent bezeichnet werden kann, ${ }^{10}$ spricht ebenfalls nichts dagegen, den Begriff der Freiheit auf tierisches Verhalten anzuwenden, von dem wir annehmen, dass es ohne die Regulation durch Vernunft, Einsicht und Wille auskommt. Gesetzt, man versteht Freiheit als den (spontanen und nicht gewaltsam behinderten) Vollzug der Eigenständigkeit, kann man sie auch auf andere Lebewesen übertragen, obgleich eindeutige Hinweise auf die Wirksamkeit eigener Einsicht und eigenen Willens fehlen: Auch ein nur seinen spontanen Regungen, seinem eigenen Verhaltensprogramm oder den gattungsspezifischen Regeln seiner sozialen Einheit folgendes Lebewesen kann als „frei“ gelten, solange es nicht in der Falle sitzt, in einen Käfig gesperrt ist oder an der Leine laufen muss.

Wenn im Kontext unablässig mit- und gegeneinander auftretender Kräfte, die strenge kausale Gesetze vollstrecken, so etwas wie Leben auftreten kann, ja, wenn es unter ihren Bedingungen sogar möglich ist, von Überraschung, Zufall oder fehlender Berechenbarkeit zu sprechen, dann kann es auch kein Problem sein, eben hier Freiheit für möglich zu halten, ohne von einem Widerspruch zur kausalen Determiniertheit auszugehen. Man muss nur in Erinnerung haben, dass die Erfahrung von Freiheit im vollen Sinn des Wortes nur im Kontext des menschlichen Handelns möglich ist. Von hier aus kann der Mensch dann Vorformen seiner Freiheit im Verhalten der Tiere erkennen.

9 Hinzukommen Handlungen, die durch einen schweren organischen Defekt, wie zum Beispiel bei einer Sucht, hervorgerufen werden.

10 Etwa dann, wenn jemand seinen Intuitionen folgt und sich von den Gründen anderer nicht beirren lässt. Auch dann handelt er im üblichen Verständnis „,frei“. 
Sogar die - ihrer spezifischen Natur entsprechende - Hinwendung der Pflanzen zum Licht kann der Mensch als eine Vorstufe seiner eigenen Freiheit wahrnehmen, zumal es auch hier die Möglichkeit gibt, die Pflanzen zu reglementieren. Wie die Erfahrung der Freiheit der Möglichkeit einer gewaltsamen Behinderung korrespondiert, so ist auch die Rede von der „,freien“ Natur daran gebunden, dass der Mensch ihr „Fesseln“ anlegen kann.

11. Natürliche Freiheit. Das Leben ist der Bereich der Natur, an dem wir auch „,innerlich“ Anteil nehmen. Denn ihm gehören wir nicht nur äußerlich, sondern gänzlich zu - sowohl in unserem Stoffwechsel als auch in unseren Empfindungen und Gefühlen. Die Anteilnahme erlaubt uns zu sagen, ob sich unter den als gegeben beobachteten Bedingungen etwas nach eigenen Kräften ungehindert entwickeln und bewegen kann. In dieser - Erkenntnis immer schon voraussetzenden - Anteilnahme können wir dann sagen, dass sich der Fluss nicht mehr ,ungehindert“ durch die Niederungen schlängelt, sondern durch Dämme in ein festes Bett ,gezwängt“ ist. Wir sehen nicht ohne Beklemmung auf das in Reih und Glied gesetzte Obst im Spalier, bedauern die mit Maulkorb oder Trense disziplinierten Tiere oder beschleunigen unversehens, wenn wir einen Viehtransporter zu überholen haben.

In allen diesen Fällen haben wir eine Vorstellung vom natürlichen Bewegungsverlauf. Er erscheint uns zwangsläufig als „frei“, sobald wir ihn mit dem Verhalten vergleichen, das durch äußere Einwirkung erzwungen wird. Was der naturbelassenen Bewegung ausdrücklich entgegensteht, erscheint uns als gewaltsamer Eingriff, als Behinderung oder Zwang, als künstlich und eben damit nicht als „frei“. Dem entspricht die bereits erwähnte Definition der Freiheit in Spinozas Ethik: „Dasjenige Ding heißt frei, das aus der bloßen Notwendigkeit seiner Natur da ist und allein von sich zum Handeln bestimmt wird; notwendig aber oder vielmehr gezwungen (necessaria autem, vel potius coacta) dasjenige, was von einem anderen bestimmt wird, auf gewisse und bestimmte Weise zu sein und zu wirken.“11

Die Pointe von Spinozas Axiom liegt in der Verschränkung von Notwendigkeit und Freiheit. Frei ist das, was sich nach seinem eigenen Gesetz bewegt. Wir könnten, in Anlehnung an die Terminologie Immanuel Kants (dessen Freiheitsbegriff dem Spinozas nicht widerspricht), ${ }^{12}$ auch von der „Selbstorganisation“ des lebendigen

11 Teil I, 7. Definition. - Die Parallele zur Definition Jean Bodins ist offenkundig.

12 Vgl. dazu Gerhardt, Volker: Menschheit in meiner Person. Exposé zu einer Theorie des exemplarischen Handelns. In: Byrd, B. Sharon \& Jan C. Joerden (Hg.), Jahrbuch für Recht und Ethik/Annual Review of Law and Ethics, Band 14 (2006), Berlin, S. 1-10. 
Wesens sprechen. ${ }^{13}$ Frei ist demnach das, was sich nach eigenen Gesetzen selbst organisiert.

Wie nahe sich in diesem Verständnis Natur und Freiheit kommen, hat übrigens bereits ein antiker Denker vor Augen geführt. Wenn Lukrez die Natur als dasjenige definiert, was ,selber, spontan alle Dinge ganz aus sich heraus vollführt“ (ipsa sua per se sponte omnia dis agere expers), begreift er sie als eine kosmische Selbstorganisation, die sich jedem einzelnen Lebewesen mitteilt und die ihren höchsten Ausdruck im Verhalten des vernunftgeleiteten Menschen, vornehmlich natürlich des philosophierenden Weisen findet. ${ }^{14}$

Das unter Anleitung der modernen Biologie entstandene Verständnis der lebendigen Natur als einer sich in zahllosen Populationen individuell entfaltenden Selbstorganisation bringt die überlieferten Formen von Natur und Freiheit einander beträchtlich näher, als dies unter dem Paradigma der Physik möglich war. Nunmehr erscheint es immerhin als denkbar, eine Naturgeschichte der Freiheit zu entwerfen, die der Kulturgeschichte der eigentlichen, der selbstbewussten Freiheit des Menschen zugrunde liegt.

13 In seiner dritten Kritik, der Kritik der Urteilskraft von 1790, hat Kant eine ingeniöse Theorie des Lebens entworfen. Mit ihr hoffte er, den lange gesuchten Übergang von der mechanischen zur dynamischen Naturtheorie zu finden. Demnach beurteilen wir alles Lebendige als einen Fall von individueller Selbstorganisation im Prozess einer sich in und durch die Individuen vermehrenden Gattung. Jeden Organismus betrachten wir so, „als ob“ er im strukturellen Aufbau wie auch im Gang seiner prozessualen Entwicklung eigenen (und damit „freien“) Zwecken folgte. Die lebendigen Zwecke kommen unserer eigenen Vernunft in der Selbstbewegung organischer Wesen entgegen. In ihnen zeigt sich die innere Einheit der Natur, für deren Erkenntnis wir nicht mehr benötigen als das Selbstbewusstsein unserer eigenen Freiheit. Diese Freiheit erfahren wir in der Selbstbewegung aus eigener Kraft, in der wir selbstbestimmten Zwecken folgen, so dass wir darin selber Mittel unserer eigenen Zwecke sind. Im Bewusstsein unserer eigenen Freiheit organisieren wir uns selbst (vgl. Kant, Immanuel: Kritik der Urteilskraft, §49; AA 5, 313).

14 Lukrez: De rerum natura, II, 1092. Die Feststellung wird in genetischer Perspektive gemacht, und bezeichnet die „befreite Natur“ (natura libera), die sich von ihren „herrischen Zwingherren“ (dominis superbis), den Göttern also, losgelöst hat und sich nun ganz aus eigenen Impulsen bewegt. Bemerkenswert ist, dass Lukrez auch den weisen Menschen mit ähnlichen Worten beschreibt, wie die von der Vormundschaft der Götter befreite Natur: „Doch der übrige Teil der Seele, verstreut durch den ganzen Körper, gehorcht und bewegt sich nach Willen und Wink des Geistes. Der ist weise für sich allein aus sich (sibi solum per se sapit), und er freut sich auch für sich, während nichts weder Körper bewegt noch das Leben." (III, 142-146) Dazu systematisch Gerhardt, Volker: Selbstbestimmung. Das Prinzip der Individualität, Stuttgart 1999, S. 180ff. 
12. Die Spontaneität der Selbstorganisation und die Vielfalt der Arten. Verstehen wir also das Lebewesen als eine in sich kohärente Einheit, die sich aus eigenem Impuls nach seinen eigenen Regeln zu bewegen vermag: Niemand braucht anzunehmen, dass diese Regeln im Widerspruch zu den Gesetzen stehen, nach denen nicht nur die umgebende, sondern auch die den Organismus durch und durch tragende Natur verfährt. Die Kausalität gilt außen und innen. Sie ist überall anzutreffen, wo einzelne Ereignisse in nahtloser zeitlicher Sukzession aufeinander folgen. In seiner physischen Konstitution macht der Organismus keine Sprünge. Das gilt selbst für jene Fälle, in denen er selber springt.

Nehmen wir das täglich milliardenfach vorkommende Ereignis des Sprungs, ganz gleich ob er bei Flöhen, Fröschen, Spatzen, Delphinen oder Menschenkindern vorkommt. Es ist möglich, weil die Schwerkraft, die mitwirkenden Elemente und die eingesetzten Kräfte ihn ausführbar machen. Er ist aber auch möglich, weil ihn die körperliche Konstitution des Lebewesens erlaubt. Er muss innerhalb des Sets von Regeln liegen, die ein artspezifisches Verhalten möglich machen. Wie konnte es, strikte Kausalität vorausgesetzt, überhaupt dazu kommen, dass einige Arten springen oder hüpfen, andere Arten aber nicht?

Der Evolutionstheoretiker wird um eine Antwort nicht verlegen sein: Der von Anfang an auf einzelne Organismen aufgeteilte Prozess des Lebens war nicht überall den gleichen Bedingungen ausgesetzt. Also haben sich die Individuen nach den lokalen und epochalen Konditionen differenziert, sodass sich im Laufe von Jahrmillionen die Regeln der Selbstorganisation der Lebewesen geändert und zu hoch differenten Spezies geführt haben. Schon darin zeigt sich eine „Freiheit“ der Natur, die in der Korrespondenz zu spezifischen Umweltbedingungen ganz unterschiedlich reagieren konnte und im Laufe der Entwicklung eine unübersehbare Vielfalt einzelner Arten hervorgebracht hat. Viele können springen, viele aber auch nicht. Die Natur hat sich die Freiheit genommen, die Baupläne der Organismen mal so und mal so anzulegen, obgleich der strenge Mechanismus der Kausalität (so wie ihn sich die Deterministen denken) eigentlich eine universelle Uniformität nahelegt.

13. Die Individualität der Reaktionen. Die gleichen konstitutionellen Regeln, nach denen sich die Individuen einer Art verhalten, haben ebenfalls nicht die Folge, durchweg zu den gleichen Verhaltensweisen zu führen. Es ist nicht so, dass alle Flöhe gleichzeitig in der gleichen Weise springen. Sie springen, jeder für sich und zwar an Ort und Stelle, also dort, wo sie durch ihren eigenen Impuls zu einer bestimmten Reaktion auf die äußeren Reize veranlasst werden.

Die Differenzierung der Individuen potenziert die Differenzierung der Ausgangslagen um ein Vielfaches, und niemand wundert sich, dass in der Nacht zwar alle 
Katzen grau, in ihrem Sprung nach dem Opfer (an Ort und Stelle) aber vollkommen einzigartig sind. Ich kenne niemanden, der unter Hinweis auf die durchgängige kausale Determination, die Vielfalt in den organischen Vollzügen bestreiten würde. Mir ist auch niemand bekannt, der die These vertritt, es könne das von so vielen $\mathrm{Zu}$ fällen geprägte und so viele Überraschungen bietende Leben gar nicht geben, weil alles kausal determiniert ist.

Nun könnte jemand behaupten, dass die Vielfalt in den konkreten Verhaltensweisen die gewachsene Vielfalt der Individuen exakt und unverrückbar wiedergibt. Dass es zwar die Differenz der Arten und der Individuen gibt, dass aber jedes Individuum genötigt ist, auf jeden eindeutig bestimmten Reiz aus seiner Umwelt immer in exakt derselben Weise zu reagieren. Doch auch das ist offensichtlich nicht der Fall. Denn die in allen Nächten graue Katze muss sich vor dem Mauseloch nicht immer in exakt derselben Weise verhalten: In einem Fall schaut der Mausekopf genügend weit hervor, so dass sie springt, im andern Fall aber unterlässt sie es, weil die Erfolgsaussichten weniger günstig sind. Wie kommt es zu diesem Unterschied? Mal springt sie und mal springt sie nicht?

Auch hier wäre vermutlich niemand um eine Auskunft verlegen: Katzen, nicht anders als Flöhe, Frösche, Spatzen oder Delphine, verhalten sich individuell zu den teils vorgefundenen, teils selbst geschaffenen Situationen. Es hängt von den jeweils an Ort und Stelle gegebenen Konditionen im Inneren und im Äußeren des Organismus ab, wie er reagiert. Ist der Bedürfnisdruck hoch, springt die Katze womöglich schon bei der kleinsten Regung vor dem Mauseloch; ist es in der Nacht schon die dritte günstige Gelegenheit, die ihr schon zweimal zu einem Erfolg verholfen hat, kann es sein, dass sie aus Trägheit gar nicht springt, obgleich sich die Beute schon in ganzer Länge vorgewagt hat. Wie erklärt uns der Biologe den Unterschied?

Ich vermute, er verweist auf den Gesamtzustand des Organismus: Hunger setzt die Schwellenwerte herab, Sättigung setzt sie herauf. Doch das müssen nicht die einzigen Faktoren sein: Hat das Tier Junge zu versorgen, kommen zusätzliche Reaktionsfaktoren hinzu; die Jahreszeit, die Witterung, die Lichtverhältnisse und die Windrichtung können eine Rolle spielen; außerdem muss man die Häufigkeit der Jagdgelegenheit und des Jagderfolgs nicht bloß in einer Nacht in Rechnung stellen.

Die Frage ist nur, wie, wo und wodurch die verschiedenen Einflussfaktoren verrechnet werden. Wie, wo und wodurch wird entschieden, zu welchem Verhalten es kommt? Es versteht sich heute von selbst, dass man hier nur zu Antworten gelangt, wenn man die Funktionsweise des Gehirns einer Untersuchung unterzieht. Die Gehirnvorgänge können uns vermutlich die wichtigsten Auskünfte über die Reaktionsformen der höher organisierten Lebewesen geben. Deshalb ist die Neurophysiologie gleichsam die Königs- oder die Zentralratsdisziplin der Verhaltensbiologie. Aber sie 
kommt zu überzeugenden Einsichten nur, wenn sie bei allen Erklärungen den Tatbestand einbezieht, dass sich der Organismus als Ganzer zu verhalten hat.

Auch wenn das Gehirn sich wesentlich mit sich selbst beschäftigt, so geschieht das doch nur, um die Leistungsfähigkeit für den ganzen Organismus zu erhöhen. Und stets hängt es vom Gesamtzustand des Organismus ab, welches Verhalten er bei welchen Umweltreizen zeigt. Die Individualität des Organismus und die Situativität seiner spezifischen Reaktionen sind durch die Einheit des Organismus bestimmt. Das ist schon deshalb kein gewagter Schluss, weil jedes Verhalten eines Organismus immer schon ein Ausdruck des ganzen Organismus ist. Jeder Reiz wird dem ganzen Organismus vermittelt; und in jedem Zucken eines Gliedes, erst recht in jedem (den ganzen Organismus mitnehmenden) Sprung, reagiert der Organismus ganz. Auch wenn sich zunächst nur die Schnurrbarthaare der Katze sträuben: Es ist die ganze Katze, die zum Sprung ansetzt.

14. Reflexive Mechanismen. Sobald wir vom Ganzen eines Organismus sprechen, haben wir die lineare Erklärung nach dem Kausalschema hinter uns gelassen. Alle Einzelvorgänge mögen dem direkten Kausalnexus entsprechen, das Ganze aber reagiert als System, das durch seinen spezifischen Konnex spezifischer Regeln seine eigene Gesetzmäßigkeit im Umgang mit äußeren Reizen hat. In Relation zu den separaten externen und internen Vorgängen nimmt sich das System, wenn ich so sagen darf, die Freiheit, sich so zu verhalten, wie es ihm entspricht.

Natürlich gehen wir nicht so weit, jedem System „Freiheit“ nach Art der menschlichen Selbsterfahrung zuzusprechen. Andererseits aber käme wohl niemand auf die Idee, dem Organismus die Möglichkeiten zu hoch spezialisierten und das heißt zugleich: zu hoch individualisierten Verhaltsformen abzusprechen. Da jedes Individuum einzigartig ist und im Gang seines Lebens durch die Summierung von Eindrücken, Erfahrungen und Leistungen nichts von dieser Individualität verliert, ist auch nicht anzunehmen, dass sich die Besonderheit des Systems verliert, in dem das Individuum seine Einheit hat.

Die Lebensgeschichte findet in der körperlichen Beschaffenheit des Organismus ihren Niederschlag. Aber entscheidend sowohl für den Organismus wie auch für den Betrachter ist, dass sich gegebene und gewachsene Individualität im Verhalten des Organismus dokumentieren. Das Verhalten ist Ausdruck des ganzen Systems, als das wir den Organismus begreifen. Folglich sind die Exposition und die Expression des Ganzen durch es selbst vermittelt. - Wem diese Ausdrucksweise zu geheimnisvoll erscheint, der kann den (immerhin technisch simulierbaren) Begriff des reflexiven Mechanismus verwenden, der hinreichend deutlich anzeigt, dass sich das Verhalten eines Organismus nicht auf lineare Kausalrelationen reduzieren lässt. 
Also haben wir in den reflexiven Mechanismen, von denen in Biologie, Soziologie und Informatik die Rede ist, eine reale Form physischer Wirksamkeit in nicht linearkausaler Form. ${ }^{15}$ Dazu brauchen wir den Bezug auf die individuell verstandene Einheit eines lebendigen Wesens. Dieser Bezug liegt uns nicht zuletzt deshalb so nahe, weil wir uns selbst als lebendige Einheit begreifen. Entsprechendes gilt für soziale Körperschaften, in denen wir uns selbst als ein lebendiger Teil einer lebendigen Einheit verstehen. Dieses Verständnis kann durch die kausalmechanische Reduktion schon deshalb nicht bestritten werden, weil es auch noch den Prozess der Erkenntnis trägt, der zu kausalmechanischen Reduktionismen führt.

15. Freiheit als Selbstbestimmung aus eigenen Gründen. Um wenigstens bis an die Schwelle eines systematischen Modells zur Rekonstruktion der Evolution der Freiheit zu gelangen, brauchen wir nur (ich sage „nur“) an die Stelle der sprungbereiten Katze einen seiner selbst bewussten Menschen zu setzen. Es genügt dann schon, das menschliche Bewusstsein als eine Instanz der kommunikativ verfügbaren Realität zu fassen, ${ }^{16}$ die es ermöglicht, Verhalten wenigstens partiell zu kontrollieren und zu koordinieren, um zu sehen, dass sich durch das Bewusstsein die Zahl der systembedingten Einflussfaktoren exponentiell erhöht.

Ohne das Bewusstsein in seinen Leistungen zu überschätzen, kann man sagen, dass es die Selbstreferenz des Systems erheblich steigert. Im bewussten Zustand werden sachhaltige Momente des Wissens, die Ausdruck einer mit anderen bewusst geteilten Wirklichkeit sind, in den Komplex der Selbstorganisation einbezogen. Der zum Sprung bereite Mensch wartet auf den vereinbarten Anruf, um sich endlich auf den Weg in den Kreissaal zu machen. Er hat noch vor Augen, wie es bei der Geburt des ersten Kindes war. Deshalb wartet er schließlich auch nicht länger, sondern gehorcht seiner inneren Unruhe und fährt schon vorher los. Niemand zwingt ihn dazu. Seine Frau hat erst gestern noch einmal betont, er müsse nicht dabei sein, wenn er nicht wolle. Doch die Erinnerung an das erste Mal, die Vermutung, dass es der Frau, trotz der gespielten Gelassenheit, wichtig ist, wenn er dabei ist, nötigen ihn, den Anruf gar nicht erst abzuwarten.

15 Das ist der Prozess, um den es bei der Freiheit geht. Wenn reflexive Mechanismen mit der Naturkausalität vereinbar sind (woran offenbar niemand zweifelt), dann braucht es auch zwischen Leben und Kausalität keinen Widerspruch zu geben - ganz gleich wie man die Kausalität zu fassen sucht. Wenn aber zwischen Leben und physikalischer Ordnung kein Widerspruch besteht, braucht es ihn auch zwischen Freiheit und Kausalität nicht zu geben. Das ist die schlichte These, die aus meiner Überlegung folgt.

16 Gerhardt, Volker: Die Instanz der Realität. In: Merkur, Heft 677/678 (2005), S. 273-283. 
Hier reagiert ein Organismus aufgrund der Regeln, die zur naturalen und kulturellen Konstitution seiner Spezies gehören, auf eine Unzahl von äußeren und inneren Gegebenheiten an Ort und Stelle, und er tut dies ganz, also als eine Einheit, die durch die reflexiven Mechanismen der Erinnerung und der bewussten Absicht individuell derart komplex werden, dass es (um nicht von einem Gott zu sprechen) nur einem Supercomputer möglich wäre, den ganzheitlichen Effekt der ganzheitlichen Verrechnung aller Faktoren, die zum Verhalten des Menschen führen, aktuell zu erfassen.

Diese Verrechnung im Ganzen eines Organismus zu einem Ganzen des Verhaltens, das überdies in einer kommunikativ erschlossenen sozio-kulturellen Einheit verständlich sein muss, kürzen wir ab und sprechen von „Freiheit“, wenn das menschliche Individuum die für sein eigenes Verständnis wichtigen Momente zu überschauen glaubt und sich ihnen im eigenen Verhalten überlässt. Das kann es nicht, wenn es durch ein anderes Individuum ausdrücklich an der Ausführung des Verhaltens gehindert wird. Ist das aber nicht der Fall und kann es Auskunft über die vermutlich ausschlaggebenden Momente des eigenen Verhaltens geben, kann es die von ihm selbst in seiner bewusst erfahrenen Ganzheit beglaubigten Faktoren „Gründe“ nennen. Wenn er dies tut, sind wir überzeugt, dass der Mensch im Bewusstsein der Freiheit gehandelt hat. Dabei hat er nur die Unendlichkeit der individuellen Bedingungen, die durch seine eigene Konstitution, durch die Besonderheit in Raum und Zeit, die Spezifika seiner Kultur und die der gerade gegebenen sozialen Konstellation so abgekürzt, wie das für die sachhaltige Verständigung selbstbewusster Wesen üblich ist.

Wenn es möglich sein soll, unter diesen Bedingungen im Rekurs auf die von bewussten menschlichen Wesen beanspruchte intentionale Steuerung ihres Verhaltens sinnvoll zu handeln, ist es unverändert zweckmäßig, sich unter Berufung auf den Begriff der Freiheit zu verständigen. Dabei brauchen wir nicht zu unterstellen, dass im Menschen die Natur in zwei Teile zerfällt. Also müssen wir auch nicht erst Kompatibilisten werden, um sinnvoll von Freiheit zu sprechen. Wenn wir den Unterschied zwischen einem Teil und einem Ganzen beachten, verfügen wir auch schon über die ganze Kunst des Perspektivismus, mit dem uns ein Teil der Philosophen die Freiheit verständlich macht.

In meinen Augen genügt es, wenn wir nur ernsthaft versuchen, die Eigenart des Lebens zu verstehen. Denn der Physikalismus scheitert nicht erst am Geist, sondern bereits an den Prozessen des Lebens. Und im Vergleich von Freiheit und Leben ist Leben allemal das größere Problem. 
16. Post scriptum im Jahr der Geisteswissenschaften. Die vorgetragene Überlegung ${ }^{17}$ ist von einer einheitlichen Konzeption der Natur bestimmt. „Natur“ ist der reichhaltigste Begriff für die Realität, in der wir uns befinden und die wir selber sind. Je nach Kontext mag es angemessen sein, von „Sein“ oder „Welt“, von „All“ oder „Wirklichkeit“" zu sprechen - verzichtbar sind alle diese Begriffe nicht, auch wenn man sich um größte Distanz zur Metaphysik bemüht. Aber sobald man einen gehaltvollen und zugleich anschaulichen Ausdruck für das Ganze, in dem wir sind und zu dem wir selbst gehören, finden will, bleibt als adäquater Begriff nur der der Natur zurück.

Die Unumgänglichkeit dieses Begriffs zeigt sich auch darin, dass Natur alternativlos ist. Dem in der Regel rein grammatisch konstituierten Sein steht das Nichts gegenüber, dem eher sensualistisch-pragmatisch konzipierten Wirklichen lässt sich das Unwirkliche der Illusion entgegenstellen; zur emphatisch-praktisch angelegten Welt sind Gegenwelten denkbar. Die Negationen des lebensweltlich begründeten, aber szientifisch erprobten Naturbegriffs jedoch führen stets auf etwas, das innerhalb der Natur verbleibt. Die Unnatur ist eine Perversion, die längst als zur Natur gehörend begriffen wird. Und alle Varianten des Nicht-Natürlichen, vom Künstlichen der Kunst, über die Technik, die Gesellschaft bis hin zur Zivilisation, zur Kultur und zum Geist, sind Differenzierungen innerhalb der Natur. Sie lassen sich sämtlich als Entwicklungsformen der Natur begreifen.

Die einzige mir bekannte Ausnahme, ist der Gottesbegriff, sofern er mit der Unterstellung einhergeht, Gott stehe als Schöpfer der Natur notwendig außerhalb von ihr. Einen solchen Gott aber dürfte man nicht als „lebendig“, vermutlich auch nicht als „allmächtig“ bezeichnen. Deshalb haben die Philosophen Gott auch stets als „erste Ursache" der Natur konzipiert, die als solche zur Natur gehört. Nicht selten haben sie das Göttliche mit der Natur gleichgesetzt. Solange es Naturwissenschaften im heutigen Verständnis gibt, ist das aber nicht zu empfehlen, so großartig die Formel Spinozas Deus sive natura auch ist.

Sehen wir vom Göttlichen ab, kann eigentlich nur die Stellung des Geistes fraglich sein. Tatsächlich wird niemand behaupten wollen, der Geist gehöre zur Natur wie Stock oder Stein. Er dürfte auch vom Leib und von dessen Ausdruck, somit auch von der Sprache (!) unterschieden sein. ${ }^{18}$ Man wird ihn ferner nicht mit dem Gehalt einer Mitteilung oder mit dem Zweck einer Handlung identifizieren wollen. Geist ist das Einzigartige, das ein Individuum unmittelbar mit dem begriffenen Sachverhalt

17 Vgl. meinen Beitrag in: Heilinger, Jan-Christoph (Hg.), Naturgeschichte der Freiheit, Berlin, New York: de Gruyter, 2007, S. 457-479.

18 Dazu Gerhardt, Volker: Im Jahr des Geistes. Philosophiekolumne. In: Merkur, Heft 696 (2007), S. 339-346. 
verbindet. Deshalb scheint der Geist auch außerhalb von Raum und Zeit zu sein. Aber muss er deshalb als das ganz Andere der Natur erscheinen - zumal er ohne den direkten Bezug zwischen dem Ich und der von ihm bewusst erfassten Sache gar nichts bedeutet?

Nehmen wir das Beispiel der Technik: Sie ist von den gegebenen Dingen, die man am Strand auflesen oder von Bäumen pflücken kann, wohl unterschieden - allein dadurch, dass sie durch eine zielgerichtete Tätigkeit des Menschen entsteht. Diese Tätigkeit geht von einem Naturwesen aus, dass sich die Beschwerlichkeit seiner natürlichen Bewegungen durch den Einsatz kraftsparender Mittel erleichtert. So kommt es zu einem Werkzeug, das die Erwartungen nur erfüllt, sofern es nach Art eines natürlichen Gegenstandes einsetzbar ist: Unabhängig davon, ob es sich um einen Faustkeil, eine Uhr oder um ein Notebook handelt, ist und bleibt es ein physisches Ding, das nach physikalischen Gesetzen wirksam ist. In dieser physischen Wirksamkeit dient das Werkzeug einem Wesen, das selbst Natur sein muss, um die Wirkung seiner Technik erfahren und angemessen schätzen zu können. Wie weit diese Verbindung zwischen Natur und Technik gehen kann, zeigt sich daran, dass der Mensch seinen eigenen Körper als Werkzeug benutzen kann - etwa dann, wenn er jemandem einen Faustschlag versetzt oder sich über die Reling hängt, um das Kentern des Bootes zu verhindern.

Also gehört die Technik zur Natur; sie ist Natur, denn anders könnte sie als Technik gar nicht zur Geltung kommen. Die besondere Genese der Technik, die deutlich von der der anderen Naturgegenstände unterschieden ist, gibt somit keinen Anlass, sie aus der Natur zu eliminieren.

Übertragen wir das Beispiel auf den Geist, lässt sich mit nahezu gleicher Anschaulichkeit zeigen, dass der Geist mit seinen der Technik durchaus vergleichbaren Leistungen des Begreifens, des Erinnerns, des Schließens oder des Verstehens, in enger Verbindung mit den physischen Gegebenheiten stehen muss, um überhaupt als Geist wirksam zu sein. Wir haben auch hier den paradoxen Tatbestand, dass der Geist qua Geist nur erkennbar oder benennbar ist, sofern er sich aus Natur auf Natur bezieht und dabei natürliche Folgen zeitigt. Mindestens insofern gehört er zur Natur.

Das größte Rätsel ist, wie es zum Anlass, zum Anstoß, zum Impuls des Geistes kommt und natürlich, wie seine unmittelbare Präsenz in seinen Leistungen zu verstehen ist. Wie bei der Technik, die ihre Besonderheit wesentlich in ihrer Genese hat, liegt auch das Eigentümliche des Geistes in der Spontaneität, in der er auftritt, und in der Unmittelbarkeit, in der er, alle Techniken überspringend, anderen verständlich ist. Dabei ist er wesentlich eigener Geist. Er kann sich also nur in der Abgrenzung von denen verstehen, die ihm in ihrem Geist unmittelbar zugänglich sind.

Hier tritt die Parallele zwischen Geist und Freiheit wohl am deutlichsten hervor. Wollte man dieser Verbindung nachgehen, hätte man mit Blick auf den Geist zu 
sagen, dass auch er - im Vergleich mit der Freiheit - das größere Problem darstellt, weil die Freiheit uns auf das Problem des Lebens führt.

Also haben wir zu klären, wie sich der Geist zum Leben verhält. Im Leben aber, das ihn zeitlich und leiblich bedingt, stellt er die Unmittelbarkeit von Beziehungen her, die Menschen untereinander benötigen, um sich eindeutig auf ein und denselben Sachverhalt zu beziehen. Ohne diese Unmittelbarkeit wäre sachhaltige Verständigung über Personen und Dinge nicht möglich. Es gäbe die mit dem Geist ursprünglich verknüpfte Sphäre eines allgemeinen Zugangs zu den Sachverhalten nicht. Damit entfiele der Raum des Bewusstseins, der, so paradox es klingt, ein öffentlicher ist.

Es ist dieser öffentliche Raum des Bewusstseins, in dem die Individuen im Verständnis von Sachverhalten unmittelbar miteinander verbunden sind. In diesem Raum entsteht die Kultur, in der die Natur des Menschen ihre spezifische Form erhält. Wenn das der Geist bewirkt, muss es als abwegig erscheinen, ihn selbst als etwas anzusehen, das nicht zur Natur gehört.

Es spricht vielmehr alles dafür, dass der Geist, wie die Freiheit, ein Ausdruck des Lebens ist. Wir brauchten uns nur die Mühe zu machen, die Leistung des Geistes genau zu beschreiben, dann würde er sich uns in so vielen Besonderheiten zeigen, dass es nichtssagend wäre, in ihm lediglich die Wirkung einer einzigen Ursache zu sehen. Der Reduktionismus, der im Naturalismus der Gegenwart immer noch dominiert, stellt sich nicht nur den Geist und das Leben, sondern auch die Natur als viel zu einfach vor. 